\title{
El modelo de reactancia-indefensión de Wortman y Brehm desde la perspectiva de la psicofisiología social
}

\author{
I. FERNÁNDEZ JIMÉNEZ DE CISNEROS y J. M. LEÓN \\ RUBIO \\ Universidad de Sevilla
}

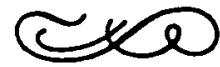

\section{Resumen}

Este estudio fue diseñado para poner a prueba la bipótesis de reactancia-indefensión de Wortman y Brehm (según la cual los efectos producidos por la incontrolabilidad están mediados por la duración del evento incontrolable) en relación al funcionamiento corporal. Para ello, se eligió como principal variable dependiente el control de la respuesta electromiográfica de la musculatura frontal. Se utilizó un diseño factorial 3 (expectativa de control alta, baja y nula) $\times 2$ (entrenamiento en incontrolabilidad corto y largo). Asimismo, se incluyeron medidas sobre "locus de control", estilo atribucional, percepción y estrategias de control. Los resultados obtenidos confirman parcialmente la hipótesis establecida y son discutidos dentro del ámbito de la Psicofisiología Social.

Palabras clave: Reactancia. Indefensión.

Dirección del autor: Facultad de Filosofía y CC Educación. Avda. S. Francisco Javier, s/n. 41005 Sevilla.

Agradecimientos: Una primera versión de este artículo fue presentada al II Congreso Nacional de Psicología Social, celebrado en Alicante los días 6, 7 y 8 de abril de 1988.

\section{A socio-Psychophysiological View of Wortman \& Brehm's Model on Reactance and Learned Helplessness}

\section{Abstract}

The purpose of the present study was to test Wortman and Brebm's bypothesis on reactance-learned belplessness phenomena (the effects produced by uncontrollability are mediated by the duration of the uncontrollable event) in relation to corporal functioning. The EMG response of the frontal muscle was the main dependent variable, and a 3 (bigh, low, and null control expectative) $\times 2$ (short and large duration of uncontrollability training) factorial design was employed. Measures of locus of control, attributional style, control perception and control strategy were obtained, as well. Results of EMG response revealed an statistically significant interaction between expectative of control and duration of uncontrollability training, and the results of the control perception revealed a main effect of the second factor. These findings are discussed in terms of the Social Psychophysiology.

Keywords: Reactance. Learned Helplessness. 
En el estudio de la conducta humana ha sido frecuente el enfrentamiento entre lo biológico y lo social, con posturas reduccionistas en uno y en otro enfoque. Aunque a lo largo de este siglo ha habido varios intentos para integrarlos —entre ellos cabe citar el esfuerzo de Schachter y Singer (Schachter y Singer, 1962; Schachter, 1964); para aunar los determinantes cognitivos, fisiológicos y sociales de la emoción- ello no adquirirá entidad hasta finales de los 70, con la labor desplegada por Cacioppo y Petty (1983) en torno a la sistematización de la Psicofisiología Social, término utilizado para designar aquel campo de investigación interdisciplinar centrado en a) el estudio de los efectos de la interacción social sobre las respuestas fisiológicas reales o percibidas, y b) en cómo ciertos estados fisiológicos (por ejemplo el «arousal») están implicados en la determinación de la conducta social.

El presente trabajo, en consecuencia, se ha centrado en uno de los tópicos que más atención está recibiendo actualmente dentro de nuestra disciplina, cual es el del «control», y especialmente los efectos nocivos que su ausencia puede producir (ver, por ejemplo, Rhodewalt y Strube, 1985; Taylor, Lichmant y Wood, 1984; Glass y Carver, 1980). En concreto, las teorías de la «reactancia psicológica» (Brehm, 1966, 1981) y de la «indefensión aprendida" (Seligman, 1975; Abramson, Seligman y Teasdale, 1978; y Miller y Norman, 1979) estudian qué reacciones se producen ante la incontrolabilidad y tratan de ofrecer una explicación de ella. Aunque a primera vista ambas puedan parecer muy dispares, Wortman y Brehm (1975) las integraron sobre la base de las expectativas de control y la duración del entrenamiento en incontrolabilidad. De tal manera que ante una situación incontrolable, la reactancia precedería a la indefensión en los individuos que esperan ejercer control, y mientras mayores fuesen sus expectativas de control más tiempo tardarían en volverse indefensos. Por otro lado, al aumentar la exposición a la incontrolabilidad los individuos perderían sus expectativas de control y se tornarían indefensos.

El propósito de este estudio ha sido extender las predicciones establecidas en dicha estructura de análisis al control de las respuestas fisiológicas, y específicamente al de la actividad eléctrica de la musculatura estriada, medida por medio de la electromiografía. Las razones para la elección de dicha actividad fueron: a) la relativa facilidad con que puede ser registrada, b) la ausencia casi completa de suposiciones respecto a la evaluación y análisis de los potenciales eléctricos musculares, c) el conocimiento preciso que se posee de la naturaleza cualitativa y cuantitativa del control voluntario que el ser humano puede ejercer sobre dicha actividad, y d) el que se hayan obtenido relaciones significativas entre estas respuestas del sistema muscular esquelético y otras propias de otros sistemas de respuestas biơlógicas (León Rubio, 1986). A todo lo cual, habría que añadir la existencia de cierta evidencia acerca de que el control de la respuesta electromiográfica se deteriora bajo situaciones de incontrolabilidad (Carlson y Feld, 1981).

Para ello, se formuló la bipótesis de que los efectos producidos por la administración de resultados incontrolables (falso «feedback» del electromiograma integrado -EMG-I- de la musculatura frontal) estarían mediados por dos factores: tipo de expectativa de control inducida (éxito vs. fracaso) y duración de la exposición a la situación de incontrolabilidad (en- 
trenamiento corto vs. largo). De manera que los sujetos expuestos a la condición incontrolable de fracaso controlarían más sus respuestas electromiográficas (efecto de reactancia), y al incrementarse el periodo de exposición a los resultados incontrolables se deterioraría dicho control (efecto de indefensión).

\section{METODO}

\section{Sujetos}

De una población total de 60 estudiantes de Psicología de la Facultad de Filosofía y Ciencias de la Educación de la Universidad de Sevilla que respondieron voluntariamente a la demanda de participación en un experimento sobre «Registros psicofisiológicos", se seleccionó una muestra de 30 sujetos sobre la base de los siguientes criterios:

- No poseer antecedentes psicopatológicos.

- No manifestar problemas de tensión muscular.

- No estar recibiendo tratamiento farmacológico continuado.

- No poseer experiencia en técnicas de relajación y/o meditación.

- No haber participado previamente en experimentos similares.

- No padecer trastornos de audición y/o visión o dermatológicos en las zonas de piel en que se situarían los electrodos.

De los 30 sujetos seleccionados, 21 eran mujeres y 9 varones, con edades comprendidas entre 18 y 24 años, y una edad media de 20,2 años.

Todos ellos desconocían el objetivo del experimento y antes de su inicio fueron entrevistados individualmente por el experimentador con un doble propósito:

a) Asegurar su participación en el estudio, según la disponibilidad de tiempo de cada uno de ellos.

b) Establecer un "pacto de silencio" con los sujetos, a fin de que no comentasen el experimento con sus compañeros.

Los datos recogidos en dichas entrevistas individuales que tuvieron una duración aproximada de 30 minutos, no dieron lugar a ningún cambio en la composición de la muestra seleccionada.

Posteriormente, los sujetos seleccionados fueron asignados aleatoriamente y de forma proporcional a los diferentes grupos resultantes de la combinación de los dos factores considerados en el diseño de esta investigación que seguidamente se describe.

\section{Diseño experimental}

Para poner a prueba la hipótesis central de la presente investigación, se utilizó un diseño factorial intergrupos $3 \times 2$, donde el primer factor «expectativa de control» tuvo los niveles alto, bajo y nulo, y el segundo factor "duración del tratamiento" los niveles corto y largo.

La variable dependiente (VD) fue el grado de control alcanzado por los sujetos en la sesión de prueba, medido a partir del nivel de reducción del 
electromiograma integrado en comparación con sus valores basales. Para lo cual, se utilizaron dos parámetros:

a) La "magnitud de reducción del electromiograma», consistente en el valor resultante de restar al valor promedio de la línea base el valor promedio del electromiograma durante los ensayos de «biofeedback".

b) La "proporción de reducción del electromiograma", consistente en el valor resultante de dividir el valor promedio del electromiograma durante los ensayos de "biofeedback" por el valor promedio de éste durante la línea base (para el lector no familiarizado con estos conceptos puede resultar útil la lectura de Basmajian, 1976; y Burish y Horn, 1979).

\section{Procedimiento}

Una semana antes de someter a los sujetos a las dos sesiones de laboratorio que se efectuaron, fueron evaluados a lo largo de una sesión de 45 minutos de duración mediante la aplicación de las siguientes pruebas: Escala de Internalismo-Externalismo de Rotter (1966), y Cuestionario de Estilo Atribucional de Peterson et al. (1982).

Posteriormente, cada sujeto recibió en días consecutivos dos sesiones experimentales que fueron efectuadas en un laboratorio que reunía las condiciones acústicas, de iluminación y temperatura, adecuadas para el propósito del estudio.

El desarrollo operativo de ambas sesiones fue el siguiente:

\section{SESION 1}

Esta sesión tenía por objetivo la manipulación de las expectativas de los sujetos mediante su exposición a resultados (de fracaso o éxito) incontrolables, antes de ser entrenados en «biofeedback».

Dicha sesión constó de cuatro períodos:

\section{a) Período de preparación y habituación}

Para la operación de preparación del equipo de registro y colocación de los electrodos sobre la musculatura frontal se siguió el procedimiento habitual descrito en Vila (1981), al tiempo que se facilitaban las siguientes instrucciones escritas:

"Vamos a colocar unos electrodos sobre su frente para registrar una determinada respuesta. En ningún momento va a recibir ningún estímulo nocivo (como por ejemplo, un choque eléctrico), puede estar seguro de que NO resulta doloroso en absoluto. Lo único que tiene que hacer es sentarse lo más cómodamente posible y descansar tranquilamente con los ojos cerrados cuando se le indique. Habrá varios períodos de registro, que se le indicarán por el oscurecimiento de la luz. Cuando la luz tenga toda su intensidad puede abrir los ojos. Estire las piernas, procure no moverse y póngase lo más cómodo posible.»

Una vez colocados los electrodos y administradas las instrucciones, se ponía en funcionamiento el equipo de registro sobre el que se realizaban las siguientes operaciones: 
- Selección de la escala de sensibilidad del amplificador electromiográfico. En nuestro caso, de las tres escalas disponibles con un alcance respectivo de 20,200 y 2.000 microvoltios pico-pico (uVp.p.), fue seleccionada la escala de $20 \mathrm{uVp}$.p., es decir, la de máxima sensibilidad.

- Selección del tiempo de lectura del promediador digital del que se tomaron las medidas del EMG frontal. El tiempo de lectura elegido fue de 30 segundos.

Realizadas estas operaciones y con el fin de comprobar el buen funcionamiento del equipo de registro y facilitar la habituación del sujeto a la situación, se dejaron transcurrir un par de minutos en los que no se tomó ninguna medida del EMG.

\section{b) Período de registro de línea base}

Tras comprobar el buen funcionamiento del equipo, y recordarle al sujeto que permaneciera quieto y con los ojos cerrados, se efectuaron dos ensayos de registro de línea base de 8 minutos de duración, con un período interensayos de 1 minuto.

\section{c) Período de administración de resultados incontrolables}

Según el grupo al que fueron asignados los sujetos, en este período se procedió del siguiente modo:

\section{Grupo de falso «feedback» de éxito}

Los sujetos asignados a este grupo fueron sometidos a un procedimiento ideado para hacerles creer que estaban logrando un resultado positivo en la tarea de reducción de los niveles del EMG frontal. Dicho procedimiento consistió en la manipulación por parte del experimentador de la escala de ganancia del amplificador de "feedback», según una secuencia fija pre-establecida que dio lugar a la disminución progresiva del «feedback» acústico que a través de auriculares se le proporcionó a los sujetos y que previamente se había asociado con la reducción de sus niveles electromiográficos mediante las siguientes instrucciones:

«El propósito de esta investigación es analizar con detenimiento, la relación existente entre ciertas respuestas fisiológicas y otras habilidades específicas. Las personas que mejor controlan las respuestas en estudio poseen una serie de habilidades que resultan especialmente adaptativas en la vida actual, siendo éste el motivo que más nos interesa en este trabajo. Vamos a revisar la colocación de los electrodos y, como ya sabe NO va a recibir ningún estímulo doloroso. Su tarea específica consiste en disminuir la intensidad del sonido que escuchará y que corresponde a su actividad biológica. Es decir, a mayor actividad biológica mayor intensidad del sonido; la disminución del sonido le indicará que su actividad biológica ha disminuido apropiadamente. Tenemos que advertirle que no debe moverse ni tensar sus músculos, puesto que el aparato sólo puede registrar un determinado nivel de su señal biológica. Si tensa mucho su músculo frontal; la señal que escuchará no corresponderá con su actividad biológica.»

A través del amplificador de "feedback» se procesó una señal eléctrica constante generada por el equipo de registro, en lugar de la señal eléctrica 
tomada en la musculatura frontal de los sujetos. Es decir, el «feedback" acústico en ningún caso estuvo en relación de contingencia con la actividad eléctrica detectada en los sujetos.

Grupo de falso "feedback» de fracaso

Los sujetos asignados a este grupo fueron sometidos a un procedimiento ideado para hacerles creer que sus esfuerzos no daban el resultado apropiado; reducir la actividad del EMG frontal. Dicho procedimiento fue idéntico al aplicado al grupo de falso "feedback» de éxito, salvo que ahora las manipulaciones del experimentador sobre la escala de ganancia del amplificador de "feedback" dieron lugar al incremento progresivo de la señal acústica de retroalimentación.

\section{Grupo control}

Los sujetos asignados a este grupo fueron expuestos a un sonido de iguales características que el "feedback» acústico que se administró en las condiciones de falso "feedback", pero en este caso el sonido aumentó y disminuyó aleatoriamente y el experimentador no sugirió que éste covariara con la actividad electromiográfica registrada en la musculatura frontal de los sujetos, a los que tampoco se les requirió la ejecución de una tarea específica. Las instrucciones que se proporcionaron en este grupo fueron:

«El propósito de este estudio es analizar la incidencia de ciertos sonidos sobre algunas respuestas biológicas. Vamos a revisar la colocación de los electrodos y, como ya sabe, $\mathrm{NO}$ va a recibir ningún estímulo doloroso. Siéntese lo más cómodo posible, descanse con los ojos cerrados y procure no prestar atención al sonido. Habrá varios períodos con sonido y otros de descanso que se le indicarán oportunamente. Estire las pieranas, procure no moverse y no tensar excesivamente sus músculos.»

La mitad de los sujetos de cada grupo fueron asignados aleatoriamente a la condición de pre-entrenamiento corto, y la otra mitad a la de largo.

- Pre-entrenamiento corto. Consistió en un bloque de dos ensayos de 8 minutos de duración cada uno, con un período interensayos de 1 minuto.

- Pre-entrenamiento largo. Consistió en dos bloques de dos ensayos cada uno. La duración de los ensayos fue de 8 minutos, siendo el periodo interensayos de 1 minuto, y el interbloques de 3 minutos.

\section{d) Período de evaluación}

Durante este período se les pasó a todos los sujetos las siguientes escalas y cuestionarios elaborados por el experimentador para este estudio: Escala de Relajación Subjetiva, Escala de Juicios de Control, Cuestionario de Atribución de Control, y Cuestionario de Estrategia de Control.

\section{SESION 2}

Esta sesión tenía por objetivo evaluar los efectos de la exposición a resultados incontrolables sobre la ejecución en la situación de "biofeedback». 


\section{1}

Dicha sesión constó de los siguientes períodos:

a) Período de registro de línea base

En este período se procedió igual que en su homónimo de la sesión anterior.

\section{b) Periodo de «biofeedback» del EMG frontal}

Durante este período se procedió del siguiente modo con todos los sujetos: El canal de entrada de la señal electromiográfica fue conectado al amplificador de «feedback» y el conmutador de selección del tipo de «feedback» fue situado en la posición negativa, de modo que concomitante con la reducción del EMG del sujeto se produjera una disminución proporcional en la intensidad de la señal acústica de "feedback", y a la inversa. Igualmente, se seleccionó una escala de sensibilidad del amplificador de «feedback» de $1 / 4$ respecto al nivel promedio basal de cada sujeto; es decir, el sonido fue eliminado por completo cuando el sujeto entrenado logró reducir 1/4 su EMG basal. Cada vez que ocurría esto, el experimentador balanceaba la señal tomando ahora como valor basal el registrado en ese preciso momento.

Las instrucciones facilitadas a los sujetos al inicio de este período fueron las siguientes:

«Ahora la tarea consiste en tratar de relajarse lo más posible pero con ayuda del aparato. Escuchará un sonido que corresponde con su actividad biológica. A mayor intensidad del sonido mayor tensión, y a menor intensidad menos tensión. Por tanto, mientras más relajado esté menor será la intensidad del sonido. Ha de tratar de mantener el sonido lo más bajo que le sea posible o de apagarlo. La dificultad de esto se irá incrementando gradualmente, a medida que se vaya relajando. Habrá varios ensayos y períodos de descanso que se indicarán oportunamente. Cierre los ojos, estire las piernas y relájese.»

\section{c) Período de evaluación}

En este período todos los sujetos completaron las siguientes escalas y cuestionarios: Escala Internalismo-Externalismo de Rotter (1966), Escala de Relajación Subjetiva, Escala de Juicios de Control, Cuestionario de Atribución de Control, y Cuestionario de Estrategia de Control.

Las medidas del EMG registradas durante los períodos de línea base, de administración de resultados incontrolables, y de «biofeedback», fueron transformadas en un valor promedio para cada período y expresadas en uVp.p. (mocrovoltios pico-pico).

Con estos valores promedio, y a fin de valorar las diferencias entre los distintos grupos respecto al control del EMG, se calcularon para cada sujeto en ambas sesiones dos parámetros: la magnitud y la proporción del cambio.

Para calcular la «magnitud del cambio» en la primera sesión, se restó al valor de la línea base el valor promedio del período de administración de resultados incontrolables. En cuanto a la segunda sesión, la magnitud del cambio se determinó sustrayendo del valor del período de línea base el valor del período de «biofeedback». 
Cundro I

Resumen de procedimiento

SESION EVALUACION. Aproximadamente 45 minutos de duración.

\section{SESION EXPERIMENTAL 1}

PERIODO DE PREPARACION Y HABITUACION. Aproximadamente cinco minutos de duración.

PERIODO DE LINEA BASE. Dos ensayos 8 minutos de duración, con un período interensayos de 1 minuto.

PERIODO DE ADMINISTRACION DE RESULTADOS INCONTROLABLES

- INDUCCION DE EXPECTATIVAS DE EXITO: mediante la administración de falso "feedback" del EMG-I diseñado para hacer creer al S. que estaba logrando el éxito en la tarea de reducir los niveles de su EMG-I.

- INDUCCION DE EXPECTATIVAS DE FRACASO: ídem del grupo anterior salvo que en este caso el $S$. fue inducido a creer que estaba fracasando en el logro del objetivo de la tarea.

- CONTROL: los Ss. fueron expuestos a una señal de "feedback» de idénticas características a la de los grupos anteriores, pero que varió de forma aleatoria y sin sugerir que ésta covariase con su actividad biológica. La tarea en este grupo fue estar en reposo.

Los sujetos de cada grupo fueron asignados aleatoria y proporcionalmente a las siguientes condiciones:

- PRE-ENTRENAMIENTO CORTO: Un bloque de dos ensayos de 8 minutos de duración cada uno, con un periodo interensayos de 1 minuto.

- PRE-ENTRENAMIENTO LARGO: Dos bloques de dos ensayos de 8 minutos de duración, siendo el período interensayos de 1 minuto, y el interbloques de 3 minutos.

PERIODO DE EVALUACION. Aproximadamente 15 minutos de duración.

\section{SESION EXPERIMENTAL 2}

PERIODO DE LINEA BASE. Dos ensayos de 8 minutos de duración, con un período interensayos de 1 minuto.

PERIODO DE ENTRENAMIENTO EN BIOFEEDBACK. Tres ensayos de $8 \mathrm{mi}$ nutos de duración, siendo el período interensayos de 1 minuto.

PERIODO DE EVALUACION. Aproximadamente unos 15 minutos.

Para calcular la "proporción del cambio", el valor del período de administración de resultados incontrolables fue dividido por el valor basal de la primera sesión, y el valor promedio del período de «biofeedback» fue dividido por el valor basal de la segunda sesión.

Además de estas reducciones, de las diferentes medidas que se tomaron del EMG se eliminaron aquellas que consideramos se debían a movimientos bruscos del sujeto, al ruido eléctrico del equipo de «biofeedback», y a posibles artefactos de antena. Para ello se realizaron los siguientes cálculos:

- Movimientos bruscos del sujeto. Una vez revisados los datos electromiográficos, se eliminaron los valores que doblaban al valor anterior (Qualls y Sheehan, 1981).

- Ruido eléctrico del equipo. Antes de conectar el cable de los electrodos al equipo electrónico, se realizaron dos lecturas de igual duración que las de los ensayos experimentales. Las lecturas superiores a $0,5 \mathrm{uVp}$.p., dieron lugar a la corrección de los datos según la fórmula descrita por Alexander, White y Wallace (1977), consistente en hallar la raíz cuadrada de la sustracción: cuadrado de la am- 
plitud real del EMG menos el cuadrado del valor del ruido eléctrico del equipo electrónico.

- Posibles artefactos de antena. Se eliminaron todos los valores superiores al de la escala de sensibilidad seleccionada $(20 \mathrm{uVp}$.p).

\section{Material}

Los instrumentos y aparatos utilizados fueron los siguientes:

\section{a) Instrumentos}

1. Escala de Internalismo-Externalismo de Rotter. Se trata la traducción realizada por Pérez (1984) de la «Rotter's Internal-External Control Scale» (1966).

2. Cuestionario de Estilo Atribucional de Peterson. Se trata de de la traducción realizada por Salgado (1984) del- "Attributional Style Questionnaire» de Peterson et al. (1982).

3. Escala de Relajación Subjetiva. Se trata de una escala numerada del 0 al 100, indicando el valor 0 «nada de relajación» y el valor 100 «máxima relajación».

4. Escala de Juicios de Control. Se trata de una escala numerada del 0 al 100, indicando 0 «nada de control» y 100 máximo control.

5. Cuestionario de Atribución de Control. Se trata de un cuestionario ideado por los autores que consta de un ítem (¿por qué cree ud., que ha conseguido tal grado control?). La respuesta de los sujetos a dicho ítem es analizada y asignada a una de tres categorías: atribución de control interno, atribución de control externo, y sin diferenciar.

6. Cuestionario de Estrategia de Control. Al igual que el anterior, se trata de un cuestionario elaborado por la autora que consta de dos ítems (¿Cómo ha conseguido tal grado de control? ¿Qué ha hecho concretamente para realizar la tarea especificada en las instrucciones?). La respuesta de los sujetos a estos ítems es analizada y asignada a una de cinco categorías: Estrategia física pasiva (ejemplos de respuesta: «no mover los músculos de la cara, ni de las extremidades»; «no tragar saliva»; «no apretar la lengua contra el cielo de la boca", etc.), estrategia física activa (ejemplos de respuesta: "mantener la boca entreabierta"; "mantener los ojos cerrados"; "respirar pausadamente»; etc.), estrategia cognitiva activa (ejemplos de respuesta: "pensar en algo relajante»; «repetirme mentalmente que tengo que relajarme»; "simular los estados de conciencia del sueño»; etc.), estrategia cognitiva pasiva (ejemplo de respuesta: «tratar de no pensar en nada, con la mente en blancom), y sin especificar.

\section{b) Aparatos}

Para el registro del EMG y para proporcionar «feedback» de éste, se utilizó un equipo compacto de electromiografía, modelo MIOBACK CY 302, marca LETICA. Dicho equipo permite procesar la señal del EMG a través de un «band-pass» de 30 a $300 \mathrm{~Hz}$., y someterla a una constante de integración de $500 \mathrm{~ms}$. El equipo fue alimentado aisladamente de la red eléc- 


\section{4}

trica mediante una batería modelo LI-612, marca LETICA. La captación de la señal bioeléctrica se obtuvo mediante electrodos de superficie de plata clorada con forma de cúpula y un diámetro de $10 \mathrm{~mm}$. El contacto eléctrico se obtuvo mediante gel conductor modelo SPECTRA, marca PARKER. Para proporcionar al sujeto el "feedback» auditivo se utilizó un juego de auriculares modelo WH-807, marca WINNER. Por último, para el análisis de los datos se utilizó un ordenador modelo XT, marca IBM.

\section{RESULTADOS}

\section{Comparatividad de los grupos respecto a las variables no experimentales}

Con el objeto de comprobar si los grupos eran homogéneos respecto a las variables no experimentales (edad, sexo, «locus of control» y estilo atribucional) se efectuaron las siguientes pruebas:

a) Respecto a la variable edad, se realizó un análisis de la varianza para un plan factorial 3 (Condición: Control, fracaso y éxito) $\times 2$ (Duración: Corto y largo) que dio como resultado $\mathrm{F}(5,24)=.57$, n.s.

b) Para el caso de la variable sexo, se realizó una prueba de "Chi cuadrado» para cada factor del diseño experimental y para la interacción de ambos factores (variable "grupo»), obteniéndose para el cruce entre "grupo y sexo" Chi $(5)=5.2381$, n.s.; para el cruce entre «condición y sexo» Chi $(2)=.68 .6458, \mathrm{p}<.001$; y para el cruce entre «duración y sexo» Chi $(1)=.1587$, n.s. Dado que la variable sexo no se distribuyó homogéneamente en el factor condición, se efectuaron dos ANOVAs adicionales a fin de determinar si hubo o no diferencias significativas entre hombres y mujeres respecto a la reducción de los niveles del EMG (medidos por la magnitud y la proporción) tras el entrenamiento en «biofeedback». Dichos análisis no arrojaron diferencias estadísticamente significativas, con $\mathrm{F}$ $(1,28)=1.16$, n.s. y $\mathrm{F}(1,28)=.82$, n.s., respectivamente.

c) En cuanto a la variable «locus de control», se realizó un análisis de varianza para un plan factorial $3 \times 2$, cuyo resultado fue $F$ $(5,24)=.13$, n.s.

d) Por último, para comprobar si las dimensiones de la Escala de Estilo Atribucional se distribuyeron homogéneamente entre los diferentes grupos, se efectuaron para cada una de ellas un análisis de la varianza, para un plan factorial $3 \times 2$, cuyos resultados no fueron significativos en ninguna de ellas:

Internalidad de éxito $« \mathrm{~F} »(5,24)=0.77$.

Estabilidad de éxito $« F »(5,24)=1.82$.

Globalidad de éxito «F» $(5,24)=0.48$.

Importancia de éxito $« \mathrm{~F} »(5,24)=0.80$.

Internalidad de fracaso $« \mathrm{~F} »(5,24)=0.79$.

Estabilidad de fracaso $« F »(5,24)=0.17$.

Globalidad de fracaso $« F »(5,24)=0.56$.

Importancia de fracaso $« \mathrm{~F} »(5,24)=0.50$. 
Todos estos datos indican que los distintos grupos de nuestra investigación resultaron ser homogéneos para las diferentes variables no experimentales consideradas en la misma.

\section{Resultados relativos a los niveles electromiográficos}

Dado que las respuestas fisiológicas frecuentemente dependen de su nivel de línea base, se compararon las medidas basales del EMG de cada uno de los grupos en las dos sesiones de que constó el experimento. Dicha comparación fue realizada mediante sendos análisis de la varianza para un plan factorial $3 \times 2$, obteniéndose para la primera sesión $F(5,24)=.25$, n.s., y para la segunda $F(5,24)=.62$, n.s.

Con los datos de la variable dependiente magnitud de reducción del EMG durante la segunda sesión, se realizó un análisis de la varianza del modelo factorial $3 \times 2$, obteniéndose un valor $F(5,24)=3.04, p<.05$. Los contrastes de medias posteriores al análisis de varianza resultaron ser significativos para $\mathrm{p}<.05$ en los siguientes casos:

Control Corto-Fracaso Corto «t» $(24)=2.66$.

Control Largo-Fracaso Corto «t» $(24)=2.27$.

Fracaso Corto-Fracaso Largo «t» $(24)=2.96$.

Fracaso Corto-Exito Corto «t» $(24)=3.35$.

Fracaso Corto-Exito Largo "t» $(24)=3.13$.

$\mathrm{Al}$ igual que en el caso anterior, con los datos de la variable dependiente proporción de reducción del EMG durante la segunda sesión, se realizó un análisis de la varianza del modelo factorial $3 \times 2$, obteniéndose un valor $\mathrm{F}(5,24)=3.01, \mathrm{p}<.05$. Los contrastes de medias posteriores al análisis de varianza resultaron ser significativos para $\mathrm{p}<.05$ en los siguientes casos:

Control Corto-Fracaso Corto «t» $(24)=2.70$.

Control Largo-Fracaso Corto "t" (24) $=2.07$.

Fracaso Corto-Fracaso Largo " $"$ " $(24)=2.98$.

Fracaso Corto-Exito Corto «t» $(24)=3.23$.

Fracaso Corto-Exito Largo «t» $(24)=3.15$.

\section{Resultados relativos a la percepción del control}

Con el fin de comprobar si existían diferencias entre los grupos respecto al grado de control percibido por los sujetos al final de las segunda sesión, se realizó un análisis de la varianza del modelo factorial $3 \times 2$ que indicó la existencia de diferencias significativas respecto al factor duración, con $F(1,28)=4.56, p<.05$. El contraste de medias posterior a dicho análisis de varianza reveló un valor de «t» $(28)=2.14, \mathrm{p}<.05$.

Para comprobar si las manipulaciones experimentales ocasionaron o no, incrementos significativos en el grado de control percibido, se realizó una prueba «t» de Student con las puntuaciones obtenidas por los sujetos según el grupo al que fueron asignados. Los resultados de dicha prueba fueron los siguientes:

Grupo Control Corto "t" (4) $=0.00$ n.s.

Grupo Control Largo "t" (4) $=0.50$ n.s.

Grupo Fracaso Corto «t» $(4)=2.26 \mathrm{p}<.10$ 
Grupo Fracaso Largo «t» (4) $=4.52 \mathrm{p}<.05$

Grupo Exito Corto «t" (4) $=0.59$ n.s.

Grupo Exito Largo "t" (4) $=0.42$ n.s.

Finalmente, hay que señalar que los datos relativos a tipo de atribución de control, tipo de estrategia de control, "locus de control" y relajación subjetiva, no arrojaron resultados estadísticamente significativos.

\section{ANALISIS Y DISCUSION}

Antes de considerar los resultados más relevantes obtenidos en esta investigación, es necesario aludir a la comprobación de la homogeneidad de los diferentes grupos respecto a las variables no experimentales. Las pruebas estadísticas aplicadas revelaron que no hubo diferencias significativas respecto a tales variables. Ello sugiere que las diferencias observadas entre los grupos con respecto a las variables dependientes de este estudio no fueron debidas a las diferencias encontradas entre éstos en las distintas medidas que se tomaron de las variables no experimentales. Dicha comprobación se hacía necesaria toda vez que en la literatura pertinente se ha señalado el posible papel modulador que sobre el EMG, pueden desempeñar estas variables (respecto a la edad consultar Haynes et al., 1975; en cuanto al sexo ver O'Connel et al., 1979, Aznar, 1986; León, 1986; Romero, 1986; y por lo que respecta al "locus of control" ver Hall, 1979; y Romero, 1986).

Los planes factoriales y los contrastes de Tukey revelaron que sólo aquellos sujetos previamente expuestos a unos resultados negativos e incontrolables durante un corto lapso de tiempo, fueron capaces de disminuir significativamente su respuesta electromiográfica en comparación a los demás grupos. Es decir, mostraron, como se había predicho, el efecto de reactancia. Este hallazgo concuerda con los resultados que existen en el «corpus" bibliográfico sobre el tema (véase por ejemplo Brockner y Elkind, 1985; Brockner et al., 1983; Rhodewalt y Strube, 1985), aunque el tipo de tareas empleadas no hayan incluido el control de una respuesta biológica. De esta manera, se confirman y amplían las predicciones de la teoría de la reactancia al control de las respuestas corporales, al menos en lo que al EMG se refiere.

En lo que se refiere al fenómeno de indefensión que teóricamente se produciría al administrar los resultados incontrolables durante un lapso de tiempo más largo, los datos no son tan simples y claros como en el caso de la reactancia, e implican al menos la consideración de los tres aspectos siguientes:

a) Comparación de los grupos de fracaso corto-fracaso largo.

b) Comparación del grupo de fracaso largo con los de control.

c) Consideración de los grupos que recibieron en el pretratamiento falso «feedback» de éxito.

a) La comparación de los grupos de fracaso corto y largo, presta su apoyo al modelo de «reactancia-indefensión» formulado por Wortman y Brehm (1975). En éste se reconocía el parámetro temporal como elemento fundamental en la modulación de los efectos de la 
exposición a resultados incontrolables. Cuando los individuos son expuestos a este tipo de resultados, su respuesta inicial es la reactancia psicológica, mediante la que intentan recuperar el control y ejecutar la conducta apropiada (así, los sujetos del grupo de fracaso corto lograron disminuir sus niveles electromiográficos respecto a la línea base en comparación con los sujetos de fracaso largo). Si mediante este esfuerzo el individuo no consigue restablecer el control, entonces caerá en los síntomas de la indefensión aprendida (en este caso, los sujetos del grupo de fracaso largo no lograron controlar su EMG).

b) Aunque los sujetos del grupo de fracaso largo no consiguieron reducir significativamente sus niveles electromiográficos (más bien al contrario, los aumentaron ligeramente como indican los parámetros de magnitud y proporción utilizados en esta investigación como variables dependientes), no puede hablarse rigurosamente, y en sentido metodológico, de la inducción de indefensión, puesto que este grupo no difirió significativamente de los grupos de control. No obstante, el aumento de los niveles de tensión muscular experimentado por estos sujetos al proporcionarles «biofeedback» del EMG para su reducción, inducen a suponer que tal efecto sería plausible.

c) Los resultados obtenidos por los grupos de sujetos que recibieron falso "feedback" de éxito son interesantes de cara a la polémica establecida acerca de si el deterioro de la ejecución es debido a la incontrolabilidad o a los resultados aversivos en sí. Ambos grupos de falso «feedback» de éxito se diferenciaron significativamente del grupo de fracaso corto, pero como el de fracaso largo, tampoco se diferenciaron significativamente de los grupos de control. Un hecho curioso verbalizado por algunos sujetos pertenecientes a estos grupos, fue el de que creyeron como cierto el falso «feedback" de éxito, sin embargo, el verdadero «biofeedback» les resultaba difícilmente controlable, puesto que las estrategias «aprendidas con éxito» el primer día no resultaban operativas en la segunda sesión. Los sujetos probaban repetidamente su estrategia "originalmente positiva" y el resultado era la confusión al no obtener el éxito esperado, produciéndose lo que se ha denominado «ilusión de control» (v.g. Langer y Roth, 1965; Alloy y Abramson, 1982). Estos datos parecen ir en la línea de la tesis expuesta por Seligman de que el elemento clave para el deterioro motivacional sería la incontrolabilidad (siempre que tenga una determinada duración) y no la aversividad en sí.

En definitiva, estos resultados ponen de manifiesto la importancia de las expectativas previas de control, dado que el tipo de expectativas inducido en los sujetos va a determinar su posterior ejecución en la tarea de «biofeedback» del EMG. Asimismo, permiten confirmar parcialmente el modelo de Wortman y Brehm, ya que aunque el fenómeno de reactancia ha quedado ampliamente demostrado, no ha ocurrido lo mismo con el de indefensión, dado que el grupo de fracaso largo no se ha diferenciado significativamente de los restantes grupos. Ello pudiera sugerir la mayor complejidad del fenómeno de indefensión humana, y la implicación de otros aspectos no considerados usualmente en este tipo de estudios. 
Los datos relativos a la percepción del control, confirman los obtenidos por otros autores. Por ejemplo, Alloy y Abramson (1979), encontraron que los sujetos no depresivos detectaban precisamente la contingencia entre respuesta-resultado cuando este último ocurría infrecuentemente o era un evento negativo, pero sobrestimaban el grado de contingencia si el resultado experimental ocurría frecuentemente o era un evento positivo. En nuestro caso, los juicios de contingencia son más exactos cuando el evento es negativo (información de fracaso; ambos grupos de fracaso perciben menos control en la primera sesión que en la segunda), sin embargo, cuando el evento es positivo (información de éxito) se distorsionan las percepciones de control (los sujetos de los grupos de éxito no perciben diferencias estadísticamente significativas en el grado de control entre ambas sesiones, aunque hay una ligera tendencia a percibir un mayor control en la primera, ya que siete de los diez sujetos percibieron que controlaban más su respuesta en la primera sesión y uno que controlaba lo mismo en ambas), lo que presta apoyo empírico al modelo ofrecido por Alloy y Tabachnik (1984) acerca de la evaluación de la contingencia. En dicho modelo, se reconocen dos elementos fundamentales en la percepción del grado de covariación entre dos eventos: la información situacional de la contingencia objetiva entre los susodichos eventos, y las expectativas anteriores de la covariación entre esos elementos en cuestión. Dependiendo de ambos factores serán las atribuciones de causalidad y la contingencia percibida. Cuando las expectativas previas son débiles y la información actual es más fuerte, las percepciones del individuo reflejarán la contingencia objetiva entre los eventos (caso ocurrido en nuestro grupo de fracaso). Si las expectativas anteriores son fuertes y también lo es la información situacional, y ambas son incongruentes, el individuo se ve enfrentado al denominado «dilema cognitivo" (Abramson y Metalsky, 1981). Alloy y Tabachnik (1984), tras revisar las evidencias pertinentes, concluyen que éste suele resolverse en la dirección de las expectativas iniciales. Esto es precisamente lo ocurrido en nuestro grupo de éxito, en el que el sesgo previo interfirió la correcta apreciación de la contingencia en la sesión de «biofeedback». En suma, el control percibido en este estudio parece estar determinado por la evaluación de la contingencia y los elementos que afectan a ésta.

Respecto a las atribuciones de causalidad, el que no se haya obtenido un patrón claro y diferenciado de su mediación, puede haber sido debido a la inadecuación del cuestionario empleado, o bien a la distintividad de la situación experimental que exige una medida específica y no una general como la del ASQ.

En suma, nuestros datos apoyan parcialmente el modelo de Wortman y Brehm (1975), dadas las diferencias obtenidas entre los grupos sometidos a resultados incontrolables durante un período de tiempo corto y largo. La administración de resultados incontrolables que indicaban el fracaso del individuo, durante un período corto, produjeron el fenómeno de reactancia, siendo este grupo de sujetos el que más disminuyó sus niveles electromiográficos relativos a sus líneas bases. Hallazgo que concuerda con las predicciones de la teoría de la «reactancia psicológica». No obstante, y coincidiendo con otros estudios experimentales, no puede afirmarse taxativamente la inducción del estado de indefensión, puesto que los grupos so- 
metidos a un entrenamiento en incontrolabilidad largo, no se diferenciaron significativamente de los grupos de control.

Asimismo, nuestros datos apoyan el modelo de evaluación de contingencias propuesto por Alloy y Tabachnik (1984), puesto que el informe de percepción de control de los sujetos experimentales estuvo determinado por la información situacional de la contingencia objetiva entre los eventos, y las expectativas anteriores de la covariación entre ellos. Produciéndose un fenómeno de «ilusión de control» en lo sujetos previamente expuestos a falsos resultados de éxito.

Por último, cabe destacar que el tiempo de exposición a los resultados incontrolables se reveló junto al tipo de resultado, como otra variable relevante para la percepción del control. Ya que el grupo que más exactamente lo evaluó fue el de "fracaso largo».

En definitiva, este trabajo es una prueba más de la utilidad del enfoque de la Psicofisiología Social para el desarrollo y validación de las teorías de la Psicología Social.

\section{Referencias}

AbRAmSON, L. Y. y Alloy, L. B. (1981) Depression non-depression and cognitive illusions: A reply to Schwartz. J. of Exp. Psychol.: Gen., 110, 436-437.

Abramson, L. Y.; Seligman, M. E. P. y Teasdale, J. D. (1978). Learned helplessness in humans: Critique and reformulation. $J$. of Abnormal Psychol., 87, 49-74.

Alexander, A. B.; WhITE, P. D. y Wallace, H. M. (1977). Training and transfer of training effects in EMG biofeedback muscular relaxation. Psychopbysiology, 14, 551-559.

ALLOY, L. B. y ABRAMSON, L. Y. (1979). Judgement of contingency in depressed and non depressed students: sadder but wisser? J. of Exp. Psychol.: Gen., 108, 441-485.

ALLOY, L. B. y ABRAMSON, L. Y. (1982). Learned Helplessness, depression and the illusion of control. J. Pers. and Soc. Psychol., 42, 1114-1126.

ALLOY, L. B. y TABACHNIK, N. (1984). Assessment of covariation by humans and animals. Psychological Review, 91, 112-149.

AzNAR, R. (1986). Una evaluación experimental de la generalización de los efectos del biofeedback del EMG frontal. Tesis Doctorales y Tesinas de Licenciatura (resúmenes). Sevilla: Servicio de Publicaciones de la Universidad de Sevilla, 291-295.

Basmajian, J. V. (1976). Electrofisiología de la acción muscular. B. Aires: Médica Panamericana.

BREHM, W. (1966). A theory of psychological reactance. New York: Academic.

BReHM, S. S. y BREHM, J. W. (1981). Psychological reactance. New York: Academic.

BrockNer, J. y Elkind, M. (1985). Self-esteem and reactance. J. of Exp. Soc. Psychol., 21, 346-361.

BROCKNER, J. y ELKIND, M.; et al. (1983). The roles of self-esteem and self-consciousness in the Wortman-Brehm model of reactance and learned helplessness. J. Pers. and Soc. Psychol., 45, 199-209.

BURISH, T. G. Y HORN, P. W. (1979). An evaluation of frontal EMG as an index of general arousal. Bebavior Therapy, 10, 137-147.

Cacioppo, J. T. y PETTY, R. E. (1983). Social Psychophysiology. New York: Guilford Press.

CARLSON, J. G. y FELD, J. L. (1981). Expectancies of reinforcement control in biofeedback and cognitive performance. Biofeedback and self-regulation, 6, 79-91.

GLASS, D. C. y CARVER, J. (1980). Helplessness and the coronary-prone personality. En J. Carver y M.E.P. Seligman (ed.), Human Helplessness. New York: Academic Press.

HALL, J. (1979). Locus of control in electromiographic feedback. Am. J. Clin. Biofeedback, 2, 92-93.

HaYNES, S. N.; Moseley, D. y McGowan, W. T. (1975). Relaxation training and biofeedback in the reduction of frontalis muscle tension. Psychophysiology, 12, 547-552.

LANGER, E. J. y ROTH, J. (1965). Heads I win, tails it is chance: The illusion of control as a function of the sequence of outcomes in a purely chance task. J. Pers. Soc. Psychol., 32, 951-955.

LEÓN, J. M. (1986). Biofeedback y discriminación. En Tesis Doctorales y Tesinas de Licenciatura (resumen). Sevilla: Servicio de Publicaciones de la Universidad de Sevilla, 675-680. 
MilLER, I. W. y NORMAN, W. H. (1979). Learned helplessnesss in human: A review and attribution theory model. Psychol. Bull., 86, 93-118.

O'CONNELL, M. F. y YEATON, S. P. (1981). Generalized muscle changes during EMG relaxation training. Psychophysiology, 18, 56-61.

PETERSON, C. et al. (1982). The. attributional style questionnaire. Cognitive Therapy and Research, 6, 287-299.

Qualls, P. J. y SHEEhan, P. W. (1981). Trait-treatment interactions: Reply to Tellegen. J. Exp. Psychol.: General, 110, 227-231.

RHODEWALT, F. y STRUBE, M. J. (1985). A self-attribution-reactance model of recovery from injury in type A individuals. J. of Applied Soc. Psychol., 15, 330-344.

ROMERO, P. (1986). Efecto diferencial del biofeedback del EMG frontal en función de la variable locus of control. En Tesis Doctorales y Tesinas de Licenciatura (resúmenes). Sevilla: Servicio de Publicaciones de la Universidad de Sevilla, 303-306.

ROTTER, J. B. (1966). Generalized expectancies for internal vs. external control of reinforcement. Psychol. Monographs, 80.

Salgado, J. (1984). Desamparo aprendido: Atribución causal y juicios de similitud. Tesis doctoral inédita. Univ. de Santiago de Compostela.

SCHACHETER, S. (1964). The interaction of cognitive and physiological determinants of emotional state. Adv. Exp. Soc. Psycbol., 1, 49-80.

SCHACHETER, S. y SINGER, J. E. (1962). Cognitive, social and physiological determinants of emotional state. Psychol. Review, 69, 379-399.

Seligman, M. E. P. (1975). Helplessness. San Francisco: Freeman.

TAYLOR, S. E.; LICHMANT, R. R. y WOOD, J. W. (1984). Attributions, beliefs about control and adjustment to breat cancer. J. Pers. Soc. Psychol., 46, 489-502.

VILA, J. (1981). Sistemas psicofisiológicos de respuestas humanas. En A. Puerto (ed.), Psicofisiologia. Madrid: UNED, 55-90.

WORTMAN, C. B. y BREHM, J. W. (1975). Responses to uncontrollable outcomes: an integration of reactance theory and the learned helplessness model. Advances in experimental social Psychology, 8, 277-336. 